\title{
Evolution of the Cosmic Gas and the Relic Supernova Neutrino Background
}

\author{
R.A. Malaney \\ Canadian Institute for Theoretical Astrophysics, University of Toronto, \\ Toronto ON Canada M5S 1A7
}

(October 7, 2018)

\begin{abstract}
Using the redshift evolution of the cosmic gas, as inferred from QSO absorption line studies, we calculate the past supernova rate and the relic supernova neutrino background. Using this new technique we find the predicted relic neutrino flux at low energies to be at least an order of magnitude below previous estimates. We argue that the evolution of the cosmic gas is consistent with a large decrease in the number of early-type galaxies at redshifts $\sim 3$, and that this evolution is the source of the reduction in the predicted neutrino flux. Additional observational constraints from recent redshift surveys lead us to propose a modified model for the evolution of the cosmic gas in which significant infall at low redshift occurs. We discuss the possible relevance of our calculations to the X-ray emitting metal-enriched gas observed in the intergalactic medium.
\end{abstract}

Typeset using REVTEX 


\section{INTRODUCTION}

All available evidence seems to support the idea that the damped Ly $\alpha$ systems are the progenitors of current galaxies [1,2]. Also, measurements of the H I column densities in these systems allow the redshift-evolution of the neutral gas in the universe to be determined [1 3 ]. If, as argued by [1], the evolution of this gas is solely due to the transformation of gas into stars and vice versa (i.e. a closed box system), then a rather direct inference of the star formation rate (SFR) and chemical evolution at high redshift can be made [1]. Other studies which relax the closed box assumption, and which probe the possible influence of dust on the predicted chemical evolution, have also been carried out [四].

A principle aim of this work is to utilize the inferred evolution of the cosmic gas in order to ascertain the rate of core-collapse supernova as a function of redshift, and to use this information to determine the relic supernova neutrino background. Due to its potential to provide insight into the formation history of galaxies, the relic supernova neutrino background has been the focus of several previous studies [5 9]. Interest in this subject has been heightened due to the recent completion of the Superkamiokande neutrino detector [10, which will attempt for the first time a direct measurement of the $\mathrm{MeV}$ neutrino background.

By comparing our new predictions of the supernova rate with previous results based on present day galaxy counts, we shall attempt to provide some insight into the evolution of galaxies. Also, from comparisons with the luminosity densities obtained from the CFRS and Hubble Deep Field surveys, we shall explore the possibility that the evolution of the cosmic gas at low redshift requires modifications from a closed-box description.

In this report we will be extending the numerical calculations of the cosmological SFR and chemical evolution presented in a previous report [11]. Unlike the earlier work of [1, [4], the calculations reported here do not assume analytical solutions based on the instantaneous recycling approximation (IRA). In the work of [11] it was shown that the error in the

predicted cosmic SFR introduced by the use of the IRA was $\lesssim 20 \%$ (for most initial mass functions). However, in order to identify the cosmological initial mass function - knowledge 
of which is a prerequisite to determining the universal supernova rate - a detailed comparison of the predicted and observed abundances is required. For various reasons, this program is best carried out using numerical calculations of the type reported here. For further details on the calculations, beyond that given below, the reader is pointed to [11].

\section{THE PAST SUPERNOVA RATE AND RELIC NEUTRINOS}

The cosmological gas density $\Omega_{g}(z)$ is defined as the comoving gas mass density at a redshift $z$ in units of the present critical density $\rho_{c}$. Fig. 1 shows the inferred values of $h \Omega_{g}(z)$ for $q_{0}=0.5$ as tabulated by Storrie-Lombardi et al. [3] (the Hubble constant is given by $\left.H_{0}=h 100 \mathrm{~km} \mathrm{~s}^{-1} \mathrm{Mpc}^{-1}\right)$. The curves represent parameterizations of the data using the functional formalism outlined by Pei and Fall «4. The solid curve represents a parameterization to the most recent data (solid circles) [3], whereas the dotted curve represents a parameterization to the data of Lanzetta et al. [1]. In the calculations to follow we will adopt these two curves as a coarse indicator of the uncertainty in the gas evolution. Their use also allows us to explore how variations in $\Omega_{g}(z)$ affect our calculations. Uncertainty in $\Omega_{g}(z)$ is even more warranted if dust complicates the issue. The dashed curve of Fig. 1 indicates a possible correction to the dotted curve if observational bias introduced by dust effects are important, as suggested by [4]. Although the dust-corrected gas evolution is difficult to determine accurately, the dashed curve can be taken as an example of its potential effects. For the time being we shall ignore the incomplete dot-dashed curve of Fig.1. This does not represent any fit to the data, and is used later for illustrating the effects of very high supernova rates.

In the closed box model the evolution of $\Omega_{g}$ with redshift $z$ is described by

$$
\frac{d \Omega_{g}}{d z}=\left\{\int_{m(z)}^{m_{u p}}\left(m-m_{r}\right) \Psi\left(z_{m}^{f}\right) \Phi(m) d m-\Psi(z)\right\} \frac{d t}{d z}
$$

Here $z_{m}^{f}$ is the formation redshift at which a star of mass $m$ is returning gas back to the interstellar medium at the current redshift $z, \Psi$ is the $\operatorname{SFR}, \Phi$ is the initial mass function 
(IMF), and $m_{r}$ is the remnant stellar mass. The lower limit of integration $m(z)$ is the minimum stellar mass which can be returning gas to the system at redshift $z$. We assume a power law for the IMF viz. $\Phi(m) \propto m^{-(1+x)}$, normalized through the relation

$$
\int_{m_{\text {low }}}^{m_{u p}} m \Phi(m) d m=1
$$

where $m_{u p}=40 \mathrm{M}_{\odot}$ and $m_{\text {low }}=0.08 \mathrm{M}_{\odot}$ (experiments where we adjust the upper limit to $100 \mathrm{M}_{\odot}$, suggest factor $\sim 2$ modifications to most of our predicted observables). Assuming the cosmological constant is zero (as we will in all our calculations), the cosmic time $t$ is related to $z$ through

$$
\frac{d t}{d z}=-\left\{H_{0}(1+z)^{2} \sqrt{\left(1+\Omega_{0} z\right)}\right\}^{-1}
$$

where $\Omega_{0}=\left(\rho / \rho_{c}\right)_{0}, \rho$ being the energy density. Unless explicitly stated otherwise, we assume $\Omega_{0}=1$.

The rate of core-collapse supernova, $R_{\mathrm{SN}}$ can be determined through

$$
R_{\mathrm{SN}}=\rho_{c} \int_{m 16(z)}^{m_{u p}} \Psi\left(z_{m}^{f}\right) \Phi(m) d m+(1-k) \rho_{c} \int_{m 11(z)}^{16} \Psi\left(z_{m}^{f}\right) \Phi(m) d m
$$

where the lower limits of integration $m 16$ and $m 11$ have the same meaning as before, except they have lower limits of 16 and $11 \mathrm{M}_{\odot}$, respectively. The quantity $k$ is a small number related to the relative number of Type I supernovae (we will take $k=0.005$, see [11]). For the purposes at hand its adopted value is unimportant (eg. adopting $k=0$ introduces a negligible effect on the neutrino flux). With $\rho_{c}$ in units of $m$ per volume, and time in say Gyr, Eq. (4) gives the comoving supernova rate in dimensions of number per Gyr per volume.

Let us focus on the electron anti-neutrinos $\bar{\nu}_{e}$, which are the neutrinos Superkamiokande will attempt to detect. Given the temperature and total energy of the emitted $\bar{\nu}_{e}$ 's, and assuming a Fermi-Dirac energy distribution with zero chemical potential, the number of $\bar{\nu}_{e}$ 's of energy $q$ per unit $q, \frac{d N[q]}{d q}$, emitted by a supernova can be determined. Here, we adopt the temperature and energy values tabulated as a function of progenitor mass by Woosley 
et al. [7] to arrive at a weighted average for $\frac{d N[q]}{d q}$, which we call $\frac{d \bar{N}[q]}{d q}$. Clearly this weighted average will depend on the adopted IMF, and in the relations to follow one should take $\frac{d \bar{N}[q]}{d q}$ to be averaged over the given IMF.

One can show that the differential number flux of relic supernova $\bar{\nu}_{e}$ 's at the present epoch can be given in terms of the above quantities as

$$
\frac{d F_{\nu}}{d q}=c \int_{z}^{0} R_{\mathrm{SN}}(z) \frac{d \bar{N}\left[q^{\prime}\right]}{d q}(1+z) \frac{d t}{d z} d z
$$

where $q^{\prime}=(1+z) q$ and $c$ is the speed of light. Explicitly in terms of the SFR and IMF this becomes

$$
\frac{d F_{\nu}}{d q}=\frac{3 c H_{0}}{8 \pi G} \int_{0}^{z}\left\{\int_{m 11(z)}^{m_{u p}} \Psi\left(z_{m}^{f}\right) \Phi(m) d m\right\} \frac{d \bar{N}\left[q^{\prime}\right]}{d q}\left\{(1+z) \sqrt{\left(1+\Omega_{0} z\right)}\right\}^{-1} d z
$$

where we have substituted in for $\rho_{c}$ in order to explicitly show the linear dependence of the flux on $H_{0}$ ( $G$ is the gravitational constant). Although included in the calculations, for clarity we have dropped in Eq. (6) the term proportional to $(1-k)$.

In order to proceed, we must calculate the supernova rate as a function of redshift, which requires that we specify the cosmic IMF to be adopted. For a given evolution of the cosmic gas, the IMF can be chosen so as to correctly reproduce the observed cosmic metallicity at a particular redshift. (Many studies of the metal abundances in damped Ly $\alpha$ systems have now appeared, the most recent of which can be found in [12].) Using the evolutionary curve corresponding to the data of Lanzetta et al. [1] (dotted curve of Fig. 1) and normalizing to solar metallicity at redshift zero, the cosmic IMF is best described by a slope parameter $x=1.7$ [1]. Henceforth, for calculations in which we utilize the dotted (or dashed) curve of Fig.1, we will assume $x=1.7$. However, modeling the cosmic gas evolution from the recent data of Storrie-Lombardi et al. [3] (solid curve of Fig. 1), allows the IMF slope parameter to be reduced slightly. This is due to the reduced level of star formation predicted by this new data, which in turn requires a flatter IMF for a given level of metal production. With the new $\Omega_{g}(z)$ evolution a Salpeter IMF, $x=1.35$, in fact adequately reproduces the abundance data. Henceforth, we shall refer to a closed-box model using the $\Omega_{g}(z)$ evolution signified 
by the solid curve of Fig. 1, and an IMF slope $x=1.35$, as our standard chemical evolution model.

That our standard model accommodates the abundance data is seen more explicitly from Table 1, which lists the abundance mass fractions in the cosmic gas at various redshifts (here $Z$ is the total metallicity). As will become clearer later, there are doubts as to whether the closed box approximation at $z \lesssim 1.5$ is valid. For this reason it is best to normalize the IMF at higher redshifts, $z=2.5$ say. This redshift also has the advantage of being well sampled. Since its depletion onto grains is anticipated to be relatively small, $\mathrm{Zn}$ is widely used as a tracer of the gas metallicity. From Table 1 we find that the $\mathrm{Zn}$ abundance of our standard chemical evolution model is predicted to be approximately 0.03 solar at $z=2.5$, and is consistent with the mean $\mathrm{Zn}$ abundance given by [12,13] (see also later discussion of Fig.6). Thus, we are comfortable that this model is in reasonable agreement with the present abundance data of damped Ly $\alpha$ systems. Whether or not the other element abundances given in Table 1 represent the observed mass fractions in the cosmic gas, depends on the relative importance of grain depletion effects. Comparing directly with the abundances measured by [12], the predictions of Table 1 suggest a grain depletion factor of order three for Fe at $z=2.5$.

Using the evolutionary curves and the IMF's just described, Eqs.(1)-(4) lead to the supernova rates shown in Fig.2. The curve markings correspond to those shown in Fig. 1, except that we also show the rate predicted by a calculation in which our standard chemical evolution model is modified by the inclusion of a late infall term (long-dashed curve). This infall model will be explained in detail later. For convenience we have plotted the rate as the number of supernovae per year in a galaxy of mass $10^{11} \mathrm{M}_{\odot}$. Doing this requires that the rates shown in Fig.2 be multiplied by $0.01 / \Omega_{\text {gal }}$, where $\Omega_{\text {gal }}$ is the mass density (of ordinary matter) in galaxies relative to the critical density. Note that in defining the rates this way, the explicit dependencies on $H_{0}$ cancel out. We also note that except for the late-infall model, the predicted supernova rates at low redshift are quite low. For example, our standard chemical evolution model leads to a predicted supernova rate at $z=0$ of 
approximately one per 350 years per $10^{11} \mathrm{M}_{\odot}$ galaxy (for $\Omega_{\text {gal }}=0.007$ ). Although quite uncertain, the observationally inferred rate for a galaxy typical of the Milky Way is thought to be in the range of 1 per 30-100 years [14,15]. The theoretical supernova rate for a typical $10^{11} \mathrm{M}_{\odot}$ galaxy after averaging over all morphological galaxy types is also in the 1 per 100 years range, 10 Gyr after galaxy formation commenced [9].

One could take the predicted low supernova rate at zero redshift to indicate a breakdown in the extrapolation of the last data point toward zero redshift. This could be caused by any one of several effects occurring within the last data bin such as coarse averaging, galaxy evolution, or a breakdown of the closed box approximation (see later). Whatever the reason, we view the predicted low supernova rates at zero redshift with suspicion. To compensate for this, we adopt a procedure for some of our calculations in which we set the supernova rate to some constant rate, $r_{c}$, for $z<z_{c}$, where $z_{c}$ is the redshift at which our predicted rate falls below $r_{c}$. This constant rate is set to $r_{c}=a\left(0.01 / \Omega_{\text {gal }}\right)$ per 100 years per $10^{11} \mathrm{M}_{\odot}$ galaxy $(0.5 \leq a \leq 2)$.

Fig.3 displays the predicted neutrino spectrum for a variety of models. The solid curve represents the spectrum arising from our standard chemical evolution model (no low redshift $r_{c}$ correction). Three of the curves correspond to a modification (in the manner outlined above) to the supernova rate predicted by our standard chemical evolution model. For the short-dashed curve we have adopted a value of $r_{c}$ with $a=1$. Adjusting the constant rate, the lower (higher) dotted curve corresponds to a decrease (increase) of $r_{c}$ at low redshift by a factor two. The long-dashed curve is the prediction from our late infall model. We see that all these predicted spectra have a similar shape, peaking at an energy of $2-5 \mathrm{MeV}$. A significant fraction of the neutrinos in this peak can be attributed to supernovae occurring at $z \gtrsim 1$, whose emitted neutrinos are subsequently degraded in energy due to the expansion of the universe. The total integrated fluxes corresponding to these calculations are; $5.4 \mathrm{~cm}^{-2} \mathrm{~s}^{-1}$ (long-dashed), $3.9 \mathrm{~cm}^{-2} \mathrm{~s}^{-1}$ (high dotted), $2.6 \mathrm{~cm}^{-2} \mathrm{~s}^{-1}$ (short dash), $2.2 \mathrm{~cm}^{-2} \mathrm{~s}^{-1}$ (low dotted) and $2.0 \mathrm{~cm}^{-2} \mathrm{~s}^{-1}$ (solid).

It is instructive to compare our results with estimates of the neutrino spectrum which do 
not involve the use of the $\Omega_{g}(z)$ evolution. The dot-dashed curve of Fig.3 shows the neutrino spectrum we determine after approximating the supernova rate predictions of Totani et al. [9], and is typical of the neutrino spectra predicted by them for a wide range of cosmological models. For the time being we simply note that the peak in our neutrino spectra is reduced by over an order of magnitude compared to the calculations of [9]. Also, note that the total integrated flux corresponding this higher curve is $34 \mathrm{~cm}^{-2} \mathrm{~s}^{-1}$, typically an order of magnitude larger than the fluxes derived using $\Omega_{g}(z)$.

In using the $\Omega_{g}(z)$ evolution to determine the neutrino spectrum, the calculated flux is proportional to $H_{0}$. The curves are plotted assuming $h=0.5$. Therefore, given the present range of uncertainty in $H_{0}$, the flux levels could be twice that shown. There is also a dependence on the adopted value of $\Omega_{0}$, through Eq. (3). The spectra plotted are determined assuming $\Omega_{0}=1$. For $\Omega_{0}=0$, one finds the flux levels are reduced by approximately a factor of 3. However, lower $\Omega_{0}$ models produce fewer metals for a given IMF [11]. Therefore, in such a model one can adopt a flatter IMF in order to normalize the metallicity at some redshift. This in turn would lead to an increase in the predicted neutrino flux, essentially compensating the reduction caused by altering the cosmological model. This point illustrates the interplay between cosmological models and the cosmic IMF - one needs information on one in order to probe the other.

In Fig. 4 the calculations are repeated for the $\Omega_{g}(z)$ curve parameterizing the data of Lanzetta et al. [1], with the line-type of the curves retaining the meaning ascribed to them for Fig.3. Here the $2-5 \mathrm{MeV}$ peak is more pronounced as a consequence of the larger supernova rate at high redshift predicted by this $\Omega_{g}(z)$ evolution (essentially all the neutrinos in this peak were produced at high $z$ ). Again, relative to previous estimates, we see a deficiency in the predicted relic neutrino spectrum at low energies.

For the dust-corrected evolution (dashed curve of Fig.1) the predicted neutrino spectra are very similar to those shown in Fig. 3. This is to be expected since the predicted supernova rate derived using this $\Omega_{g}(z)$ evolution is similar to that predicted by our standard model (see Fig.2). In general, we find from numerical experiments that simple models of dust 
inclusion can affect the predicted flux levels by factors of up to 2. However, it is not clear at the present time how large the dust correction to the observed cosmic gas evolution should be (see also [16] for a discussion of gravitational lensing effects and dust). Thus, we will say little more about the influence of dust. The controversy over whether or not it plays an important role in studies of damped Ly $\alpha$ systems can be followed more in [17].

Unfortunately, due to various neutrino backgrounds in the $\mathrm{MeV}$ range (terrestrial, solar, atmospheric), the relic supernova neutrino background is detectable by Superkamiokande only in the limited range of approximately $15-40 \mathrm{MeV}$. In this range the neutrino flux is largely dominated by the most recent supernovae, and the predicted count rates in $\mathrm{Su}-$ perkamiokande are similar to those reported in [9] (typically a few events per year). Even if new detectors can go to lower thresholds, the large terrestrial background of antineutrinos from nuclear reactors seems to be an insurmountable problem 18 . This is a great pity since the most interesting part of the relic neutrino background (i.e. that directly connected to events in the early universe) will remain undetectable by earth-based neutrino detectors. In order to explore the low-energy supernova neutrinos some new indirect method needs to be developed.

Resonant absorption by ultra high-energy neutrinos on the relic supernova neutrino background is one such indirect method. This effect has previously been discussed in the context of the relic cosmic neutrinos predicted by big bang cosmology [19,20]. However, although absorption processes on the relic supernova neutrinos can occur at reasonable cosmic-ray energies, the low number abundance of the relic MeV neutrinos removes any reasonable hope for this technique unless the peaks in the neutrino spectra of Figs. 3 and 4 were orders of magnitude larger.

Hopefully, the importance of detecting relic supernova neutrinos in the $2-5 \mathrm{Mev}$ range can inspire other, more hopeful, methods of detection. However, in the absence of such methods, other probes of the supernova activity at high redshift deserve scrutiny. It is possible that cosmic-ray production at high redshift could lead to spectral features in the diffuse gamma-ray background [21]. By linking the past supernova rate with past cosmic-ray ac- 
tivity, one could conceive of detecting such a spectral feature commencing at $0.5 m_{\pi 0}\left(1+z_{f}\right)$ $\left(m_{\pi 0}=140 \mathrm{MeV}\right)$, where $z_{f}$ marks the redshift at which significant galaxy formation begins. However, the gamma-ray production from cosmic rays is maximized at $R_{S N} f(z) d t / d z$, where $f(z)$ depends on a myriad of uncertain effects such as models of cosmic-ray propagation and confinement. This coupled with the smooth rise predicted for $R_{S N}$, renders this an unpredictable method for measuring the past supernova rate. Below we shall discuss alternative techniques of probing past supernova activity, which appear more promising.

\section{EVOLUTION IN THE NUMBER OF EARLY-TYPE GALAXIES}

Let us further explore the discrepancy in the low-energy neutrino flux predicted by our calculations compared to those reported by [9]. We will argue that within the framework of [9], substantial evolution in the number of early-type galaxies would remove the discrepancy.

Totani et al. [9] determined a time-dependent supernova rate derived from models of galaxy evolution based on the population synthesis method. Deriving specific models of galactic evolution, they calculated the supernova rate in each galaxy type. Assuming a redshift at which galaxies form, and that the relative proportion of each galaxy type (as measured at the present epoch) remains constant with look-back time, a universal supernova rate was determined. From this rate the relic supernova neutrino background was determined in the same manner as outlined earlier.

Not surprisingly, the higher neutrino fluxes at low energy predicted by this alternative method can be traced directly to the supernova rates at early times (cf. our Fig. 2 with Figs.3-5 of ref. [9]). To further explore this, let us adopt a particular cosmological model and compare the different supernova rates in detail. We convert the supernova rates of [9] into redshift space, by assuming a formation redshift of $z_{f}=5$; which with $\Omega_{0}=1$ and $h=0.75$ leads to an evolutionary time of $\sim 0.3 \mathrm{Gyr}$ at $z=3.5$. At this epoch the calculations of [9] predict an average supernova rate of $\sim 1$ per year per $10^{11} \mathrm{M}_{\odot}$ galaxy. (the predicted

rate at this $z$ would be higher for lower $z_{f}$ values.) Such high supernova rates are roughly 
60 and 20 times larger than the supernova rates predicted by the solid and dotted curves, respectively, of Fig.2. Our calculations show that for supernova rates this high, the predicted gradient of the gas evolution $(d \Omega / d z)$ at $z=3.5$ would be significantly steeper than that indicated by the data. This is shown better by considering the dot-dashed $\Omega_{g}(z)$ curve of Fig.1. The gradient of this curve at $z=3.5$ corresponds to a supernova rate of 1 per year per $10^{11} \mathrm{M}_{\odot}$ galaxy, and is clearly incompatible with the data. Although for this calculation we adopted an IMF corresponding to $x=1.35$, numerical experiments in which we vary the IMF within reasonable bounds do not alter the situation in any significant way. It is evident that the relatively flat and smooth behavior of $\Omega_{g}(z)$ at high redshift severely limits the allowed supernova rate.

Galaxies which are elliptical (E) or intermediate between elliptical and spiral (S0), totally dominate the supernova rate at high redshifts if their relative abundance was equal to that at the present epoch. Therefore, if $d \Omega_{g} / d z$ remains relatively small one could conclude that the number of these galaxies must decrease with increasing redshift. Exactly what reduction in the number of E/S0 galaxies would provide compatibility between the predicted and observed slopes of $\Omega_{g}(z)$ is difficult to quantify. Clearly this would depend on the true evolution of $\Omega_{g}(z)$, and also on a rather uncertain error analyses of the presently available data. However, for the cosmological model being discussed, one can say that the number of E/S0 galaxies at $z \approx 3.5$ must be reduced by over an order of magnitude from their abundance at the present epoch, in order to provide any sort of match to the solid curve of Fig.1. Given the closed-box assumption, this implies that the number ratio of E/S0 to other galaxy types must have decreased by this same amount.

Note that $d z / d t$ decreases with decreasing redshift - this leads to two points. First, the gradient of the dot-dashed $\Omega_{g}(z)$ evolution (Fig.1) would have to be even steeper at lower redshift in order to accommodate the supernova rate of 1 per year per $10^{11} \mathrm{M}_{\odot}$ galaxy. Second, a redshift range $\delta z$ extends over longer evolutionary timescales at lower redshifts. These effects offer the hope that further sampling of the $\Omega_{g}(z)$ distribution at low redshift would eventually detect the onset of significant E/S0 formation. This would manifest itself 
as a breakdown in the smooth extrapolation assumed, and would most likely be found in the last data bin at $z \lesssim 1.5$.

In principal, the rapid gas evolution compatible with the predictions of [9] could be occurring prior to the highest observed redshift bin, and therefore remain undetected. To accommodate this one must adopt much earlier values of the formation redshift $z_{f}$, and/or a new cosmological model. Ideally one would prefer the first Gyr or so of E/S0 galactic evolution to occur at $z \gtrsim 5$, such as would be the case for a low $\Omega_{0}$ model with $h=0.5$ and $z_{f} \gtrsim 7$. If the relative number of E/S0 galaxies does not decrease with redshift, there should be a very rapid increase in $d \Omega_{g}(z) / d z$ for $z \gtrsim 5$. It remains to be seen whether this region will be explored in the future. We note, however, there is tentative evidence of a small decrease in the gas density at $z \gtrsim 3$, possibly indicating that above this redshift the largest damped Ly $\alpha$ systems have not fully formed [3]. If this decrease is confirmed, it would further strengthen the case for the presence of strong evolution in the E/S0 galaxy population.

An increase in the number of E/S0 galaxies with decreasing redshift is predicted to occur in hierarchical galaxy formation models as a consequence of merging processes. In

fact recently, based on data from the CFRS survey [22], evidence in support of such E/S0 evolution has been put forward [23]. Contrary to this, however, is the argument that the majority of the massive spheroid population formed at $z \sim 3$. Several pieces of observational evidence support this view also, not least of which is the detection of large elliptical galaxies at high redshift (see the review of [24]). It remains to be seen whether future survey statistics and numerical simulations will allow some combination of the two differing E/S0 evolutionary scenarios to be compatible with the evolution of the cosmic gas.

\section{LATE INFALL MODELS}

Based on column density distributions, it has been argued that the cosmic gas is best described by a closed-box system [1]. However, clearly it is difficult to extrapolate such 
arguments within the last data bin of the observations. We have already mentioned a possible discrepancy in our predicted supernova rates at zero redshift (or more generally the star formation rate) compared with what is inferred from observation. We wish to point out a further discrepancy at low redshift between our models and the observed data, which further suggests that closed-box models for the evolution of the cosmic gas require modification at late times.

In Fig. 5 we have plotted as the solid curve our estimated rate of new metal production, $\dot{\rho}_{z}$, assuming our standard model. The data points are determined from estimates of the global radiation flux below $3000 \AA$, which is assumed to be dominated by output from massive stars. Details of the UV emissivity to $\dot{\rho}_{z}$ conversion procedures are discussed in [25]. The solid circles are based on Hubble Deep Field data [25], whereas the open square is from ground-based data [26]. It is important to note that these represent lower limits to the inferred $\dot{\rho}_{z}$, since it is plausible that fainter and dust-obscured galaxies, not included in these estimates, are abundant at high redshift. The low redshift data (open circles) are based on the CFRS data of [27], and the current value of $\dot{\rho}_{z}$ is based on the H $\alpha$ luminosity density of the local universe [28].

The predicted $\dot{\rho}_{z}$ from our standard model shows a consistent fit with the high redshift data. Indeed, taken at face value it implies that the Hubble Deep Field data is missing little from its inventory. The $\dot{\rho}_{z}$ values predicted using the dotted curve of Fig. 1 are factors of 2 and 6 higher at $z=4$ and $z=2.75$, respectively. If the data points were to be revised upwards beyond these levels, a possible problem with our modeling of $\Omega_{g}(z)$ at high redshift would be indicated. At low redshift a discrepancy between the predicted and observed values of $\dot{\rho}_{z}$ is clearly evident. Since $\dot{\rho}_{z}$ can be written as

$$
\dot{\rho}_{z}=\rho_{c} \int_{m(z)}^{m_{u p}} m \Psi\left(z_{m}^{f}\right) \Phi(m) p\left(z_{m}^{f}\right) d m
$$

$p$ being the mass of the newly processed metals in the ejecta divide by the initial stellar mass, then the discrepancy may once again be related to a low rate of star formation at late times. There are many ways to accommodate this discrepancy, but perhaps the simplest is 
to allow for some infall of cool gas at late times. Dust-corrected models of the cosmic gas evolution which include infall that is a constant multiple of the instantaneous SFR, have been shown to be useful in this regard [29]. The long-dashed curve of Fig. 5 shows the $\dot{\rho}_{z}$ predicted from our late-infall model. Here we have simply added to our evolutionary equations an infall term $I(t)$ where

$$
I(t)=\Omega_{g}(z) \exp \left[-\left(\left|z-z_{p}\right|\right)^{n}\right] \quad \mathrm{Gyr}^{-1},
$$

where the fitting parameters $z_{p}$ and $n$ simply determine the peak of the infall and its width in redshift space, respectively. The prefactor $\Omega_{g}(z)$ assures that the infall is at significant levels at the chosen value of $z_{p}$. For the dashed curve shown in Fig. 5 we have taken $z_{p}=1$ and $n=2$, and kept the IMF of our standard model $(x=1.35)$. This particular lateinfall model is the one referred to in our earlier calculations of the supernova rate and the neutrino background (Figs.2-4). As is evident from a cursory look at the evolution equations the addition of any infall term would, for a given value of $d \Omega_{g}(z) / d z$, demand a higher level of star formation. As can be seen from Fig. 5, the additional star formation associated with the late infall clearly remedies the problem at low redshift. Also, since the infall is at insignificant levels at higher redshifts, the closed box results are reproduced at $z \gtrsim 2$.

In Fig. 6 the long-dashed curve displays the effect the infall term of Eq.(8) has on the key metallicity tracer, Zn. For this calculation we assumed the infalling gas had a metallicity of 0.1 solar (although even at this gas metallicity the increased Zn yield is almost completely determined by the increased SFR). The solid curve corresponds to our standard model and the dotted curve corresponds to the dotted curve of Fig.1. The data is from the tabulations of [12] (open circles) and [13] (solid circle). It is clear that our infall term does not spoil the consistency with the metallicity at $z \sim 2.5$. Although there appears a discrepancy at $z \sim 1$, we do not pay much credence to it because the data point here represents only three damped Ly $\alpha$ systems, and is most likely an unreliable tracer of the global weighted average. Nonetheless, the prediction of significantly higher Zn abundances than those indicated by the presently available data at $z \sim 1$, is a test of our late-infall model. 


\section{DISCUSSION}

It appears then, that a model of the cosmic gas described by an essentially closed-box system at high redshift, but possessing significant infall at lower redshifts, can accommodate the available observational data. We stress that our infall term has not been modeled on any specific large-scale structure simulation, but merely describes the general form required in order to satisfy the observational constraints. It is not at all obvious how the complicated processes which could occur at $z \sim 1$ would lead an infall resembling that described by Eq.(8). This is material for a future study, but in the meantime let us make some remarks which may be helpful.

In studies of galactic chemical evolution, gas infall has been shown to play an important role in accounting for the chemical and photometric properties of spiral galaxies, eg. 30,31. It therefore seems plausible that infall onto galaxies is a universal phenomenon. However, in the context of the global cosmic gas, it is difficult to quantify the accretion rate of cool star-forming gas onto the damped Ly $\alpha$ systems. But to satisfy our demands, it appears that this accretion must become efficient only at $z \lesssim 2$. It is important to emphasize that with regard to alterations in the SFR it is only the net infall that is important. It is likely that both inflow and outflow processes are taking place simultaneously, and that the net infall is the result of a combination of both processes.

Based on our earlier results we suggested the number abundance of E/S0 galaxies increases with time. The continuing merging processes associated with this evolution will lead to alterations in the cosmic gas. One would anticipate that the heat generated during mergers, eg. [32], would lead to an outflow of hot metal-enriched gas from the cool-gas system. Indeed, there is strong evidence from X-ray observations supporting the existence of a hot metal-enriched intergalactic medium that is correlated with E/S0 galaxies 33. In addition, if this hot gas represents a substantial fraction of the baryons (there is every indication that it is at least comparable to the baryon fraction in stars), then ultimately its existence must be accounted for in any model of the cosmic gas evolution. In our scenario the hot gas 
would have been produced recently due to the large E/S0 galaxy production occurring at low redshift. The net outflow from the cool-gas system associated with the hot gas production could account for the rapid fall off in $I(t)$ at $z<z_{p}$. Beyond this speculation, one can hope that full blown simulations, which include all the necessary input physics, will enlighten us further.

Finally, it is interesting to note the connection between the chemical evolution of the cosmic gas as studied here, and the models of Fields et al. [34]. This latter work, which outlines some of the complexities involved in modeling the merging processes of protogalactic gas clouds, attempts to account for metal-enriched intercluster gas and the most recent microlensing experiments [35]. A large remnant white-dwarf population in the halo of our own galaxy seems to be the most rational explanation of the microlensing experiments. If the same population was present in all spiral galaxies, then effects on (or constraints from) the cosmic gas may be anticipated. For example, our study shows how the gas should evolve under the influence of the very high star formation rates that presumably accompany the formation of the white dwarf population. However, if a large fraction of white dwarf formation took place at very high redshift $(\sim 10)$, as other observational constraints seem to require, then compatibility with the observed evolution of the cosmic gas may still be possible. The remnant white-dwarf population would play no role in the subsequent $\Omega_{g}(z)$ evolution, and any hot metal-enriched gas produced during the star formation process would play no role unless it cooled and re-entered the star-forming gas. Nonetheless, some contamination from the white-dwarf formation would have to exist at some level, and one could envisage a series of metallicity indicators at redshifts of $z \sim 3$ (eg. plateau like behavior). Variation in the IMF at early times - often invoked in discussions of the microlensing results - can also be probed by our calculations. Although we always assumed a constant IMF, Fig. 5 indicates that IMF's at early times with significantly fewer high-mass stars would not be consistent with the metal production at $z \sim 4$. These too are all topics worthy of further study, and they highlight once again the importance of a detailed theoretical description of the $\Omega_{g}(z)$ evolution. 


\section{CONCLUSIONS}

Based on the evolutionary history of the cosmic gas we have calculated the past corecollapse supernova rate and the relic supernova neutrino background that it gives rise to. We find our predicted neutrino flux to be substantially lower than other recent predictions - a discrepancy which is largest at lower energies. However, in the energy range accessible to Superkamiokande, $\sim 20 \mathrm{MeV}$, the neutrino flux is found to be compatible with previous estimates. This latter fact is not surprising since the rate anticipated at Superkamiokande is largely influenced by the supernova rate at $z \lesssim 1$. Novel techniques to measure the relic background neutrinos at $E \sim 5 \mathrm{MeV}$ would be of great importance as they would provide a direct probe of star formation activity at $z \gtrsim 1$.

Our predicted supernova rates have been used to provide some insight into the evolution of galaxies. The discrepancy between our predicted rates and previously reported rates, is removed if strong evolution in the population of early-type galaxies is included. Such an evolutionary trend seems more consistent with the evolution of the cosmic gas density, which appears to constrain the birth of most E/S0 galaxies to either very early $(z \gtrsim 5)$ or very late $(z \lesssim 1.5)$ epochs. We feel the latter epoch of formation to be more likely.

Although, a closed-box description for the evolution of the cosmic gas seems to be required by observations at high redshift, we suggest that an infall contribution at low redshift may be required. This late infall term could accommodate estimates of the global luminos-

ity density evolution measured by recent surveys. It remains to be seen whether detailed numerical simulations and future observation will lead to the required form of the late infall.

Finally, we have discussed possible connections between the evolution of the cool starforming cosmic gas, and the creation of the hot metal-enriched intergalactic gas detected by X-ray telescopes.

Investigations into the chemical evolution of damped Ly $\alpha$ systems offer new insights into the evolution of structure in the early universe. With an ever increasing wealth of data on these systems being gathered, rapid progress seems assured. 


\section{ACKNOWLEDGEMENTS}

I would like to thank B. Chaboyer for input related to stellar yields, M. Fall and M. West for discussions, and the anonymous referee for his comments. 


\section{REFERENCES}

[1] M. K. Lanzetta, A. M. Wolfe, and D. A. Turnshek, 1995, ApJ, 440.

[2] A. M. Wolfe, M. K. Lanzetta, C. B. Foltz and F. H. Chafee, 1995, ApJ, 454, 698.

[3] L. J. Storrie-Lombardi, R. G. McMahon and M. J.Irwin, 1996, MNRAS in press, astro$\mathrm{ph} / 9608147$.

[4] Y. C. Pei and S. M. Fall, 1995, ApJ, 454, 69.

[5] G. S. Bisnovatyi-Kogan and Z. F. Seidov, 1984, Sov. Astron., 26, 132.

[6] L. M. Krauss, S. L. Glashow and D. N. Schramm, D. N., 1984, Nature, 310, 191.

[7] S. E. Woosley, J. R. Wilson and R. Mayle, 1986, ApJ, 302, 19.

[8] T. Totani, and K. Sato, 1995, Astroparticle Phys. 3, 367.

[9] T. Totani, K. Sato and Y. Yoshii, 1995, ApJ, 1996, 460, 303.

[10] K. Nakamura, T. Kajita, M. Nakahata and A. Suzuki, 1994, in Physics and Astrophysics of Neutrinos, ed. M. Fukugita and A. Suzuki (Tokyo: Springer) 249.

[11] R. A. Malaney and B. Chaboyer, 1996, ApJ, 462, 57.

[12] L. Lu, L. W. Sargent and T. A. Barlow, 1996, ApJS, in press.

[13] M. Pettini, L. J. Smith and R. W. Hunstead, 1994, ApJ, 426, 79.

[14] S. van den Bergh, 1991, Supernovae, ed. S. E. Woosley (New York: Springer-Verlag) 711.

[15] G. A. Tammann, W. Löffler and A. Schröder, 1994, ApJS, 92, 487.

[16] M. Bartelmann and A. Loeb, 1996, ApJ, 457, 529.

[17] M. Pettini, D. L. King, L. J. Smith and R. W. Hunstead, 1996, ApJ, in press, astro$\mathrm{ph} / 9607093$. 
[18] P. O. Lagage, 1985, Nature, 316, 420.

[19] T. Weiler, 1982, Phys Rev Lett, 49, 234.

[20] S. Yoshida, 1994, Astroparticle Phys. 2, 187.

[21] J. Silk and D. N. Schramm, 1992, ApJ, 393, L9.

[22] S. J. Lilly, L. Tresse, F. Hammer, D. Crampton and O. Le Févre, 1995, ApJ, 455, 108.

[23] G. Kauffmann, S. Charlot and S. D. M. White, 1996, MNRAS in press, astroph/9605136.

[24] M. Fukugita, C. J. Hogan and P. J. E. Peebles, 1996, Nature, 381, 489.

[25] P. Madau, H. C. Ferguson, M. E. Dickinson, M. Giavalisco, C. C. Steidel and A. Fuchter, 1996, MNRAS in press (astro-ph/9607172).

[26] C. C. Steidel, M. Giavalisco, M. Pettini, M. E. Dickinson and K. L. Adelberger, 1996, ApJ, 462, L17.

[27] S. J. Lilly, O. Le Févre, F. Hammer and D. Crampton, 1996, ApJ, 460, L1.

[28] J. Gallego, J. Zamorana, A. Aragón-Salamanca and M. Rego, 1995, ApJ, 455, L1.

[29] S. M. Fall, 1996, (astro-ph/9611155).

[30] F. Matteucci and P. Francois, 1989, MNRAS, 239, 885.

[31] N. Arimoto and P. Jablonka, 1991, A\&A, 249, 374.

[32] S. D. M. White, J. F. Navarro, A. E. Evrard and C. S. Frenk, 1993, Nature, 366, 429.

[33] J. S. Mulchaey, D. S. Davies, R. J. Mushotzky and D. Burstein, 1996, ApJ, 456, 80.

[34] B. D. Fields, G. J. Mathews and D. N. Schramm, 1996, astro-ph/9604095).

[35] C. A. Alcock et al., 1996, ApJ submitted (astro-ph/9606165). 


\section{FIGURE CAPTIONS}

Figure 1: $h \Omega_{g}$ as a function of redshift for a de-acceleration parameter $q_{0}=0.5$. The solid circles are data from [3], and the open circles are from [1]. The curves are various parameterizations of the data, except the incomplete curve which is used to model very high supernova rates (see text).

Figure 2: The supernova rate as a function of redshift. The different curves correspond to the evolutionary curves of figure 1 (i.e. line markings are same), except the long-dashed curve corresponds to a model with infall. An IMF slope parameter of $x=1.35$ was used for the solid and long-dashed curves, with $x=1.7$ utilized for the two other curves. The rates shown should be multiplied by $0.01 / \Omega_{\text {gal }}$.

Figure 3: The $\bar{\nu}_{e}$ spectra at the present epoch, calculated using solid $\Omega_{g}(z)$ evolution of Fig.1, with $x=1.35$ (except for dot-dashed curve). The different curves correspond to different corrections to the supernova rate at low redshift (see text). $\Omega_{0}=1$ and $h=0.5$ are assumed.

Figure 4: The $\bar{\nu}_{e}$ spectra at the present epoch, calculated using dotted $\Omega_{g}(z)$ evolution of Fig.1, with $x=1.7$ (except for dot-dashed curve). The different curves correspond to different corrections to the supernova rate at low redshift (see text). $\Omega_{0}=1$ and $h=0.5$ are assumed.

Figure 5: The metal production density as a function of redshift. The data points are determined from [25] (closed circles), [26] (open square), [27] (open circles), and [28] (closed square). $\Omega_{0}=1$ and $h=0.5$ are assumed.

Figure 6: The Zn mass fraction in the cosmic gas for our standard model (solid curve), infall model (long-dashed curve), and model assuming the dotted $\Omega_{g}(z)$ evolution of Fig.1 (dotted curve). The data is from the tabulations given in [12] (open circles) and [13] (solid circle). $\Omega_{0}=1$ is assumed. 
TABLE 1.

Element Mass Fractions (-log)

\begin{tabular}{|c|c|c|c|c|c|c|c|c|c|c|}
\hline $\mathrm{Z}$ & $\mathrm{Z}$ & $\mathrm{C}$ & $\mathrm{N}$ & $\mathrm{O}$ & $\mathrm{Mg}$ & $\mathrm{Si}$ & $\mathrm{Ca}$ & $\mathrm{Cr}$ & $\mathrm{Fe}$ & $\mathrm{Zn}$ \\
\hline 5.0 & 4.5 & 5.7 & 6.4 & 4.7 & 6.1 & 5.8 & 6.9 & 7.6 & 5.8 & 9.0 \\
\hline 4.5 & 4.2 & 5.3 & 5.9 & 4.4 & 5.7 & 5.4 & 6.5 & 7.2 & 5.4 & 8.6 \\
\hline 4.0 & 3.8 & 5.0 & 5.5 & 4.0 & 5.4 & 5.0 & 6.2 & 6.9 & 5.1 & 8.3 \\
\hline 3.5 & 3.5 & 4.6 & 5.1 & 3.7 & 5.0 & 4.7 & 5.9 & 6.5 & 4.7 & 8.0 \\
\hline 3.0 & 3.2 & 4.3 & 4.7 & 3.4 & 4.7 & 4.4 & 5.5 & 6.2 & 4.4 & 7.7 \\
\hline 2.5 & 2.8 & 3.9 & 4.3 & 3.0 & 4.4 & 4.0 & 5.2 & 5.8 & 4.0 & 7.3 \\
\hline 2.0 & 2.5 & 3.6 & 4.0 & 2.7 & 4.1 & 3.7 & 4.9 & 5.5 & 3.7 & 7.0 \\
\hline 1.5 & 2.2 & 3.3 & 3.7 & 2.4 & 3.8 & 3.4 & 4.6 & 5.2 & 3.3 & 6.6 \\
\hline 1.0 & 1.9 & 3.0 & 3.4 & 2.2 & 3.5 & 3.1 & 4.3 & 4.9 & 3.0 & 6.1 \\
\hline
\end{tabular}

